\title{
Vladislav Nenchev
}

\section{DYNAMIC RELATIONAL MEREOTOPOLOGY: Logics for stable and unstable relations}

\begin{abstract}
In this paper we present stable and unstable versions of several well-known relations from mereotopology: part-of, overlap, underlap and contact. An intuitive semantics is given for the stable and unstable relations, describing them as dynamic counterparts of the base mereotopological relations. Stable relations are described as ones that always hold, while unstable relations hold sometimes.

A set of first-order sentences is provided to serve as axioms for the stable and unstable relations, and representation theory is developed in similar fashion to Stone's representation theory for Boolean algebras and distributive lattices. Then we present some results about the first-order predicate logic of these relations and about its quantifier-free fragment. Completeness theorems for these logics are proved, the full first-order theory is proved to be hereditary undecidable and the satisfiability problem of the quantifier-free fragment is proved to be NP-complete.
\end{abstract}

Keywords: stable and unstable relations, mereology, mereotopology, representation theory, hereditary undecidability, quantifier-free fragment.

\section{Introduction}

This paper presents the notions of stable and unstable mereotopological relations and several results for representation theory and first-order logics for them. It is an extension of previous work about stable and unstable mereological relations in [12]. These papers feature relational structures with stable and unstable variants of some relations from mereology or mereotopology. Stable and unstable are, in some sense, dynamic variants of the base relations. The intuition behind a stable relation 
corresponds to the case in which the relation always holds (i.e. the relation holds in all moments of time or in all possible situations). Respectively, the intuition behind an unstable relation corresponds to the case in which the relation holds sometimes, but not necessarily always. Stable and unstable mereotopological relations are also defined in [23] and [22], but in systems with richer language which includes the operations and constants from Boolean algebras. Here, as well as in [12], we consider language with only relations and no operations or constants. Thus, both these papers are relational generalizations of [23] and [22].

The stable and unstable mereotopological relations represent a development in the field of alternative theories of time and space. The mereotopological base of the stable and unstable relations expresses the spatial aspect of this development. Here we build upon the ideas of de Laguna [3] and Whitehead [24]. They use mereotopology as a base in order to build an alternative, "point-free" theory of geometry. The temporal aspect of the development is represented by the dynamic nature of the semantics of the corresponding spatio-temporal relations between regions. Thus, stable and unstable can be viewed as simple temporal operators, corresponding to always and sometimes. The fact that we have relations with both spacial and temporal nature also corresponds to Whitehead's idea for integrated theory of space and time, in which the spatial and temporal languages cannot be separated from one another.

\section{Spatial development - point-free space}

In this paper we use a weakened subsystem of mereotopology that has only four relations: part-of, overlap, underlap and contact (denoted $\leq, \mathrm{O}$, $\mathrm{U}$ and $\mathrm{C}$ respectively). Mereotopology is an extension of mereology with topological relations. Mereology is an ontological discipline which can be characterized shortly as a theory of "Parts and Wholes" (see [17] for more about mereology). It deals with objects identified as regions of space and the possible operations and relations between them. For instance, in mereology we can express common situations between regions in which one region is part of another, they share a common sub-region (this is an alternative definition for overlap) or the two regions combined do not exhaust the whole space (they underlap). The topological relations that we add are usually based on the topological contact. The contact relation between two regions means that they share a common point. Since points in mereotopology are not regions contact is stronger than 
overlap. For instance, it is possible that two regions are not overlapping but are in a contact and in this case they have only boundary points in common but not points from their interiors.

Mereology and mereotopology are used to build region-based theories of space (see [15, 4, 2, 21]). They are geometric theories that are not based on the primitive notions of point and line, like in classical Euclidean geometry, but on the notion of region $([24,3])$. The idea is that regions are much more natural objects that can easily be observed in reality, while points and lines are too abstract and do not have separate existence in reality. One of the most popular mereotopological systems is the Region Connection Calculus (RCC) introduced by Randell, Cui and Cohn [16]. Two RCC subsystems are the well-known EgenhoferFranzosa RCC-8 [6] and its mereological reduct RCC-5. Many logics based on them have been studied - see [11] and [25] for RCC-8 logics and [11] and [9] for RCC-5.

While mereology is an ontological system, Tarski has shown that its mathematical equivalent are complete Boolean algebras without the zero element (see [17] for details). Usually the zero element is added for simplicity of the definitions. The choice of Boolean algebras seems natural since they present in some way a point-free analogs of sets. It is now commonly accepted that the mathematical equivalent of mereotopology is the notion of contact algebra $([4,5,18,21])$. Contact algebras are systems $(\underline{B}, \mathrm{C})=(B, 0,1, .,+, *, \mathrm{C})$, where $\underline{B}$ is a Boolean algebra (. ', ' + ' and ' $*$ ' are the Boolean multiplication, addition and complement) and $C$ is a binary relation over $B$, satisfying the following conditions:

$$
\begin{aligned}
x \subset y & \Longleftrightarrow x \neq 0 \& y \neq 0, & x \subset y & \Longrightarrow y \subset x, \\
x \subset(y+z) & \Longleftrightarrow x \subset y \text { or } x \subset z, & x . y \neq 0 & \Longrightarrow x \subset y .
\end{aligned}
$$

We can give two concrete definitions (constructions) of contact algebras (see for instance [14]). Let $(X, \mathrm{R})$ be a relational system, where $\mathrm{R}$ is a reflexive and symmetric binary relation over $X$. Consider the pair $(\underline{B}, \mathrm{C})$, where $\underline{B}$ is the Boolean algebra of the subsets of $X$ and $C$ is defined for every $x, y \subseteq W$ as follows:

$$
x \subset y \quad \text { iff } \quad \exists a \in x, \exists b \in y, a \mathrm{R} b .
$$

Then $(\underline{B}, \mathrm{C})$ is a contact algebra. The other construction uses topological spaces and defines the contact algebra as the pair of the Boolean algebra of the regular closed sets of the topological space and the topological 
contact relation. Thus, contact algebras are really Boolean algebras extended with a contact relation. This corresponds to the fact that mereotopology is an extension of mereology with contact-like (topological) relations.

Note that although we work with a much simpler sub-system of mereotopology (with only four relations) this is actually more difficult because we cannot use the other relations to give us more information about the regions. This effect is further increased by the fact that we also work only with the relations and do not consider the functional operations and the constants from the language of the Boolean algebras.

In most cases, in which such point-free spatial systems are developed ([14], [12], [23], [22]), a generalization of Stone's representation theory for Boolean algebras and distributive lattices (see [1]) is developed also. The representation theory links the standard definition of these systems with their general definition. The standard definition states that the regions are certain sets of points. In this setting we use the points as primitive objects. Then we study the properties of the regions and formulate the general definition which states that the regions are just objects that satisfy the studied properties. It this general setting the regions are the primitive objects. Thus, the representation theory actually uses the regions from the general definitions to recreate points, which we call abstract points. Each abstract point actually represents the set of all regions that contain this point.

\section{Temporal development - moment-free time}

According to Whitehead in his studies of alternative theories of space and time, the theory of time cannot be separated from the theory of space and their integrated theory has to be extracted from the existing spatiotemporal relations considered as primitives. Thus we aim to extend the mereotopological relations with some temporal properties to obtain such combined spatio-temporal relations.

The current approach to that aim will be to enhance the spatial relations, so that they can express how they change over time. The construction that we will use is the following: consider a changing spatial environment and take a snapshot of it at every moment of time. Thus we have a collection of static environments, such that in every one of them we can define the usual mereotopological relations. Then we define two 
dynamic variants of the base mereotopological relations: stable variants and unstable variants.

Here is the formal description of the construction. Let $I$ be the set of all considered moments of time and let $a$ and $b$ be two spatial objects (regions). Then every object may exist or may not exist at every time moment (in the second case we will consider that the object is just the empty region). Thus every object can have a different instance at every moment. So we will consider that a history of an object is the collection of all of its instances. Thus, the histories of $a$ and $b$ will be $a=\left\langle a_{i}: i \in I\right\rangle$ and $b=\left\langle b_{i}: i \in I\right\rangle$. Let $R$ be one of the considered spatial relations. Respectively, $R$ has an instance for every time moment $i$, denoted by $R_{i}$. $a_{i} R_{i} b_{i}$ will denote the fact that the objects $a$ and $b$ are in the relation $R$ at moment $i$. Thus, for every original spatial relation $R$ we define two versions that describe its stability in the dynamic environment-stable version denoted by $R^{\forall}$ and unstable version denoted by $R^{\exists}$ :

$$
\begin{aligned}
& a R^{\forall} b \quad \text { iff } \quad \forall i \in I, a_{i} R_{i} b_{i}, \\
& a R^{\exists} b \quad \text { iff } \quad \exists i \in I, a_{i} R_{i} b_{i} \text {. }
\end{aligned}
$$

For instance $a \mathrm{C}^{\forall} b$ means that in each moment of time $a$ is in a contact with $b$, i.e. $a$ and $b$ are always in a contact. Similarly $a C^{\exists} b$ means that $a$ and $b$ are sometimes in a contact.

One possible way to apply this construction to obtain systems with stable and unstable mereotopological relations is the following: Take one static mereotopological structure (as defined in [14]) for every moment of time. Then consider the Cartesian product of the sets of regions from all static structures. The elements of this product are just the possible histories of the regions. Then for each base relation in the spatial language define its stable and unstable versions over the vectors. This will represent the standard definition for structures with stable and unstable mereotopological relations in this paper.

This method is used also in [23] and [22] to define stable and unstable variants of relations. In [23] and [22] dynamic systems are defined with Cartesian products of contact algebras. These systems include the Boolean operations in their language. In the current paper (as well as in [12]) the systems include only relations and no functional operations or constants. Relational systems for mereotopology may have some philosophical motivation which we will not discuss now. Here relational systems are preferred, because they are more suitable for defining modal 
logics as, for instance, in [11], [12] and [14]. Also the relational systems allow us to consider only a subset of the vectors from the Cartesian product, without violating the functions. This allows to make better models of natural environments with the current dynamic systems. Of course, by giving up the functions and the constants from the language, we make the representation theory more difficult.

Also, since we work only with the stable and unstable relations, the time moments are hidden in the construction of the relations. Thus, we cannot use the time directly in the language. In this sense, we can say that the developed theories are also "moment-free", as well as "pointfree". This, of course, leads to further complications in the representation theory for the dynamic structures. Now we have to recreate the moments of time (define abstract moments of time), as well as the spatial points.

Of course the above mentioned construction is one of the simplest ways for building stable and unstable versions of relations. Instead, we could use more complex expression over the set of time moments than just a single quantifier over $I$. For example, a modal approach could be used (also described in [22]) to define stability and unstability:

$$
\begin{array}{lll}
a R^{\forall} b & \text { iff } & \square(a R b), \\
a R^{\exists} b & \text { iff } & \diamond(a R b) .
\end{array}
$$

In this modal aspect the current construction corresponds to the S5 modality which means that we do not have any structured relation between the time moments. If we wish to define dynamic relations corresponding to more complex temporal operators we could use some ordering of the time moments - linear time, branching time, etc. In other words, we could use modality different from S5. The current paper covers only the S5 case.

Similar techniques for temporalizing spatial logics are described in [8], [25] and [10]. The general approach is to apply temporal operators over formulas or terms from the spatial language. In these papers several logics are described by means of this approach. They also represent development in the theories of space and time and most of them have more complex language and more expressive power than the ones presented in this paper. The difference is that the temporal and spatial operators in the language of these logics are separated, whereas the language of the stable and unstable mereological relations combines temporal operators and spacial relations into one. Thus, it is closer to Whitehead's idea that the theory of time cannot be separated from the theory of space. 


\section{Aims and structure of the paper}

There are two main goals in this paper.

The first is to develop the notions of stable and unstable mereotopological relations, their standard and general definitions and their representation theory. The standard definition is given by means of Cartesian products of static mereotopological structures. The general definition consists of presenting them as relational structures, satisfying a set of first-order axioms. We develop the representation theory through a generalization of the notions of filters and ideals (like in $[19,20,14,12]$ ). Thus we show that every model of the axioms can be isomorphically represented as a structure from the standard definition.

The second goal is to study the first-order logics of the stable and unstable mereological relations. We address several properties of these logics, such as their completeness, the hereditary undecidability of the full first-order theory and the NP-completeness of the satisfiability of its quantifier-free fragment.

The paper is structured in five sections. The first and the second sections present some results from [14] and [12] on which the current paper is based. The third section contains the definitions and the representation theory for the dynamic mereotopological relations. The fourth section contains the results about the first-order logics of the dynamic mereotopological relations. Finally, the paper ends with conclusion and an appendix section, which contain more technical proofs about the representation theory. The following standard reference books are provided: [17] for mereology and [1] for Stone representation theory.

\section{Static mereotopological structures}

This section contains some preliminary results about the static mereotopological theory. It features definitions for mereotopological structures and other notions and statements from [14]. They are used as a base to develop the dynamic mereological theory (the next section) and the dynamic mereotopological theory (the main results in this paper). The structures that we use here feature only the four relations $\leq-$ part-of, O - overlap, U - underlap, and C - contact, compared to the original definitions in [14], which include two more mereotopological relations the dual contact $\widehat{C}$ and the interior part-of $\ll$. This difference leads to a 
different definition of the relation $\mathrm{R}$ between the abstract points, which is a part of the representation theory for the static mereotopological structures. Thus, proofs will be given only for those results and notions that are changed here. The remaining part of the proofs can be found in [14].

Definition 1 . Let $(B, 0,1, .,+, *, C)$ be a contact algebra. Then we call the relational structure $\underline{W}=(W, \leq, \mathrm{O}, \mathrm{U}, \mathrm{C})$ a (static) standard mereotopological structure if $W \neq \emptyset, W \subseteq B, C$ is the contact relation and $\leq, \mathrm{O}$ and $\mathrm{U}$ are binary relations over $W$, defined as follows

$$
x \leq y \quad \text { iff } \quad x . y *=0, \quad x \bigcirc y \quad \text { iff } \quad x . y \neq 0, \quad x \cup y \quad \text { iff } \quad x+y \neq 1 .
$$

Next we give an alternative definition for the mereotopological structures, which is more suitable for the representation theories presented in this paper

Definition 2. Let $(X, \mathrm{R})$ be a relational system such that $\mathrm{R}$ is a reflexive and symmetric binary relation over $X$. Then we call the relational structure $\underline{W}=(W, \leq, \mathrm{O}, \mathrm{U}, \mathrm{C})$ a (static) relational mereotopological structure if $W \neq \emptyset, W \subseteq \mathcal{P}(X)$ and $\leq, \mathrm{O}, \mathrm{U}$ and $\mathrm{C}$ are binary relations over $W$, defined as follows

$$
\begin{aligned}
& x \leq y \quad \text { iff } \quad x \subseteq y, \quad x \bigcirc y \quad \text { iff } \quad x \cap y \neq \emptyset, \\
& x \cup y \quad \text { iff } \quad x \cup y \neq X, \quad x \subset y \quad \text { iff } \quad \exists a \in x, \exists b \in y, a \mathrm{R} b .
\end{aligned}
$$

If $W=\mathcal{P}(X)$ then we call such structure full.

This definition is equivalent to the previous one, because every such reflexive and symmetric relational system $(X, \mathrm{R})$ generates a contact algebra and every contact algebra can be represented as generated by such system (see [14]). The representation theory in [14] also allow us to give another equivalent definition of the mereotopological structures through topological spaces, but such definition will not be required here.

Lemma 1.1. Let $\underline{W}$ be either a standard or a relational mereotopological structure. Then $\underline{W}$ satisfies the following first-order conditions
(M1) $x \leq x$
(M2) $x \leq y \& y \leq z \Longrightarrow x \leq z$
(M3) $x \leq y \& y \leq x \Longrightarrow x=y$
(M4) $x \mathrm{O} y \Longrightarrow y \mathrm{O} x$
(M8) $x \mathrm{U} y \Longrightarrow y \mathrm{U} x$
(M5) $x \mathrm{O} y \Longrightarrow x \mathrm{O} x$
(M9) $x \cup y \Longrightarrow x \cup x$ 
(M6) $\quad x \mathrm{O} y \& y \leq z \Longrightarrow x \mathrm{O} z$

(M10) $x \leq y \& y \mathrm{U} z \Longrightarrow x \mathrm{U} z$

(M7) $x$ O $x$ or $x \leq y$

(M11) $y \cup y$ or $x \leq y$

(M12) $x \leq y$ or $x \mathrm{O} z$ or $y \mathrm{U} z$

(M13) $x$ O $x$ or $x \mathrm{U} x$

(C1) $x \mathrm{C} y \Longrightarrow y \mathrm{C} x$

(C3) $x \mathrm{C} y \Longrightarrow x \mathrm{O} x$

(C2) $x \mathrm{O} y \Longrightarrow x \mathrm{C} y$

(C4) $x \mathrm{C} y \& y \leq z \Longrightarrow x \mathrm{C} z$

Thus, we can give the general definition for the mereotopological structures

Definition 3. Let $\underline{W}=(W, \leq, \mathrm{O}, \mathrm{U}, \mathrm{C})$ be a relational structure, such that $W \neq \emptyset$. Then we call $\underline{W}$ a (static) mereotopological structure if it satisfies conditions (M1)-(M13) and (C1)-(C4).

The reduct $(W, \leq, \mathrm{O}, \mathrm{U})$ is called a (static) mereological structure.

In order to illustrate the use of (M1)-(M13), (C1)-(C4) we show one consequence from them, which is important later on

Lemma 1.2. The following statement holds in every mereotopological structure

$$
z \mathrm{C} t \& x \overline{\mathrm{U}} y \& z \overline{\mathrm{O}} y \& t \overline{\mathrm{O}} x \Longrightarrow x \mathrm{C} y \text {. }
$$

Proof. Suppose $z \mathrm{C} t, x \overline{\mathrm{U}} y, z \overline{\mathrm{O}} y$ and $t \overline{\mathrm{O}} x$.

From $x \overline{\mathrm{U}} y$ and $z \overline{\mathrm{O}} y$, by (M12), we get $z \leq x$. From $x \overline{\mathrm{U}} y$, by (M8), we get $y \overline{\mathrm{U}} x$. Then, by (M12), from $y \overline{\mathrm{U}} x$ and $t \overline{\mathrm{O}} x$ we obtain $t \leq y$.

From $z \mathrm{C} t$ and $t \leq y$, by $(\mathrm{C} 4)$, it follows $z \mathrm{C} y$. By (C1), from $z \mathrm{C} y$ we have $y \mathrm{C} z$. Then from $y \mathrm{C} z$ and $z \leq x$, by (C4) again, we have $y \mathrm{C} x$. Finally, by $(\mathrm{C} 1)$, from $y \mathrm{C} x$ it follows $x \mathrm{C} y$.

In the following proofs in this paper, in order to shorten the proof, we may often omit the use of (M4), (M8) or (C1). We will freely swap the arguments of $\mathrm{O}, \mathrm{U}$ or $\mathrm{C}$ when needed, without referring to (M4), (M8) or (C1), knowing that these three conditions ensure that $\mathrm{O}, \mathrm{U}$ and $\mathrm{C}$ are symmetric.

\subsection{Representation theory}

The representation theory of mereotopological structures is a generalization of Stone's technique for distributive lattices and Boolean algebras 
(see [1]). It shows that if we define the mereotopological relations with the conditions from Definition 3 then we can equivalently represent them as defined by a reflexive and symmetric relational system (Definition 2). For this purpose, given a mereotopological structure, we must recreate the points from the domain of the system and the relation $\mathrm{R}$ between them. To achieve this we need to define relative variants of the standard set-theoretic notions of (prime) filters and (prime) ideals. Namely, the prime filters will serve as abstract points of space. In essence, an abstract point is the collection of all regions, that should contain this point in the standard definition of regions.

Definition 4. Let $\underline{W}$ be a mereotopological structure and let $F \subseteq W$.

- $F$ is called an upper set if for all $x, y \in W, x \in F$ and $x \leq y$ imply $y \in F$;

- $F$ is called a filter if $F$ is an upper set and for all $x, y \in W, x \in F$ and $y \in F$ imply $x \mathrm{O} y$;

- $F$ is called a prime filter if $F$ is a filter and for all $x, y \in W, x \notin F$ and $y \notin F$ imply $x \cup y$.

We will also call a prime filter an abstract space point or just an abstract point.

Let $I \subseteq W$. Then the dual notion of a (prime) ideal is defined as follows:

- $I$ is called a lower set if for all $x, y \in W, y \in I$ and $x \leq y$ imply $x \in I$;

- $I$ is called an ideal if $I$ is a lower set and for all $x, y \in W, x \in I$ and $y \in I$ imply $x \mathrm{U} y$;

- $I$ is called a prime ideal if $I$ is an ideal and for all $x, y \in W, x \notin I$ and $y \notin I$ imply $x$ O $y$.

Notation. The collection of the abstract points for $\underline{W}$ will be denoted with $A P(\underline{W})$.

Definition 5. Let $\underline{W}$ be a mereotopological structure. We define the relation $\mathrm{R}$ over the filters of this structure as follows

$$
F \mathrm{R} G \quad \text { iff } \quad \forall x \in F, \forall y \in G, x \subset y .
$$

This definition is different from the one given in [14]. The difference is that here $\mathrm{R}$ is defined only with $\mathrm{C}$, while in [14] the definition involves also the relations $\widehat{C}$ and $\ll$. Still, $R$ is a reflexive and symmetric relation. The symmetry follows from the symmetry of $\mathrm{C}$. The reflexivity is checked as 
follows: take an arbitrary filter $F$ and $x, y \in F$; then from the definition of a filter we have that $x \mathrm{O} y$ and so, by (C2), $x \mathrm{C} y$.

$\mathrm{R}$ is a relation over the filters and, ultimately, it is a relation over the prime filters (i.e. the abstract points). Thus $(A P(\underline{W}), \mathrm{R})$ is the needed reflexive and symmetric relational system for the representation.

Proposition 1.1 (Static Characterization). Let $\underline{W}=(W, \leq, \mathrm{O}, \mathrm{U}, \mathrm{C})$ be a mereoto-pological structure and $x, y \in W$. Then

$(\leq) \quad x \leq y \quad$ iff $\quad \forall F \in A P(\underline{W}), x \in F \Longrightarrow y \in F$;

(O) $\quad x O y$ iff $\exists F \in A P(\underline{W}), x \in F \& y \in F$;

(U) $\quad x \cup y$ iff $\exists F \in A P(\underline{W}), x \notin F \& y \notin F$;

(C) $\quad x \mathrm{C} y \quad$ iff $\quad \exists F, G \in A P(\underline{W}), x \in F \& y \in G \& F \mathrm{R} G$.

This proposition is proved by constructing specific prime filters (abstract points) for the different cases. Here we introduce some notations that allow us to build filters and ideals.

Notation. Let $\underline{W}$ be a mereotopological structure and $x \in W$. Then we introduce the following notations

$$
[x)=\{y \mid y \in W \text { and } x \leq y\},(x]=\{y \mid y \in W \text { and } y \leq x\} .
$$

$[x)$ is the least upper set containing $x$ and $(x]$ is the least lower set containing $x$. These properties are proved in [14], as well as the following ones

LEMma 1.3. If $\underline{W}$ is a mereotopological structure and $x, y \in W$ then

1. $x \mathrm{O} y$ iff $[x) \cup[y)$ is a filter;

2. $x \cup y$ iff $(x] \cup(y]$ is an ideal;

3. $x \mathrm{C} y$ iff $[x) \mathrm{R}[y)$.

If $G$ is an upper set and $J$ is a lower set then

4. $G \cap J \neq \emptyset \quad$ iff $\quad \exists x \in G, \exists y \in J, x \leq y$.

If $F$ is a filter and $I$ is an ideal then

5. $F \cup[x)$ is a filter iff $x \bigcirc x$ and $\forall y \in F, x \bigcirc y$;

6. $I \cup(x]$ is an ideal iff $x \cup x$ and $\forall y \in I, x \cup y$;

7. If $F \cup I=W$ then $F$ is also a prime filter (an abstract point) and $I$ is also a prime ideal;

8. If $\left\{A_{i}\right\}$ is a non-empty chain of filters (ideals), linearly ordered by set-inclusion, then $\bigcup A_{i}$ is also a filter (ideal). 
With these constructions we can build specific filters and ideals. But for the characterization we need prime filters (and prime ideals). So here follow a couple of lemmas (Separation Lemma and R-extension Lemma), that allow us to expand filters and ideals to their prime counterparts. Separation Lemma is already proved in [14]. R-extension Lemma is also proved in [14] under the name $\rho$-extension Lemma. But because of the difference in the definitions of $\mathrm{R}$ we will have to repeat the proof with the new definition, to make sure that the lemma still holds.

Lemma 1.4 (Separation Lemma). Let $\underline{W}$ be a mereotopological structure and $F^{\prime}$ and $I^{\prime}$ be a filter and an ideal, such that $F^{\prime} \cap I^{\prime}=\emptyset$. Then there exist a prime filter (an abstract point) $F$ and a prime ideal $I$ so that

$$
F^{\prime} \subseteq F, I^{\prime} \subseteq I \text { and } F \cap I=\emptyset .
$$

The lemma, given below, is required for the proof of R-extension Lemma

LEMma 1.5. Let $\underline{W}$ be a mereotopological structure and $F^{\prime}$ and $G^{\prime}$ be filters and $I^{\prime}$ and $J^{\prime}$ be ideals, such that $F^{\prime} \mathrm{R} G^{\prime}, F^{\prime} \cap I^{\prime}=\emptyset$ and $G^{\prime} \cap J^{\prime}=$ $\emptyset$. Let $x$ be an element from structure such that $x \notin F^{\prime}$ and $x \notin I^{\prime}$. Then at least one of the following two conditions hold:

$$
F^{\prime} \cup[x) \text { is a filter, }\left(F^{\prime} \cup[x)\right) \cap I^{\prime}=\emptyset \text { and }\left(F^{\prime} \cup[x)\right) \mathrm{R} G^{\prime}
$$

$$
I^{\prime} \cup(x] \text { is an ideal and } F \cap\left(I^{\prime} \cup(x]\right)=\emptyset
$$

Proof. Since $x \notin F^{\prime}$ then for every $y \in F^{\prime}$ we have $y \not \leq x$. Similarly $x \not \leq y$ for every $y \in I^{\prime}$. Then, by Lemma 1.3 (?), it follows that both $F \cap(x]=\emptyset$ and $[x) \cap I^{\prime}=\emptyset$ hold. Lets consider several cases:

1) Assume that $x \cup x$ and for every $y \in I^{\prime}, x \cup y$. Then, by Lemma $1.3(1.3), I^{\prime} \cup(x]$ is an ideal and so (b) holds.

If the conditions from the first case are not true, then we have two possibilities: that $x \overline{\mathrm{U}} x$ or that there is $y \in I^{\prime}, x \overline{\mathrm{U}} y$.

2) $x \overline{\mathrm{U}} x$ holds. Then by (M13) $x \mathrm{O} x$. Take arbitrary $z \in F^{\prime}$. Then $z \mathrm{O} z$. Since $x \overline{\mathrm{U}} x$, then by (M11) we get $z \leq x$. Thus from $z \mathrm{O} z$ and $z \leq x$, by (M6), we have $z \mathrm{O} x$. So, by Lemma $1.3(1.3), F^{\prime} \cup[x)$ is a filter.

The same reasoning shows that for every $t \in G^{\prime}, t \mathrm{O} x$ and so, by (C2), $t \mathrm{C} x$. Take $v \in[x)$ and $t \in G^{\prime}$. Then, by (C4), we have $t \mathrm{C} v$ and so $G^{\prime} \mathrm{R}\left[x\right.$ ). Thus $\left(F^{\prime} \cup[x)\right) \mathrm{R} G^{\prime}$ and so (a) holds.

3) There is $y \in I^{\prime}$, such that $x \overline{\mathrm{U}} y$. This case is similar to the previous. $y \in I^{\prime}$ so $x \not \leq y$ and, by (M7), $x$ O $x$. If $z \in F^{\prime}$ then, because $F^{\prime} \cap I^{\prime}=\emptyset$ and by Lemma $1.3(?), z \not \leq y$. Thus from $z \not \leq y$ and $x \overline{\mathrm{U}} y$, by 
(M12), we get $z \mathrm{O} x$. Analogously if $t \in G^{\prime}$ then $t \mathrm{O} x$ and $t \mathrm{C} x$. The rest is the same as in the previous case and finally we have that (a) holds.

Lemma 1.6 (R-extension Lemma). Let $\underline{W}$ be a mereotopological structure and $F^{\prime}$ and $G^{\prime}$ be filters and $I^{\prime}$ and $J^{\prime}$ be ideals, such that $F^{\prime} \mathrm{R}$ $G^{\prime}, F^{\prime} \cap I^{\prime}=\emptyset$ and $G^{\prime} \cap J^{\prime}=\emptyset$. Then there exist prime filters (abstract points) $F$ and $G$ and prime ideals $I$ and $J$ so that (for the definition of R see Definition 5)

$$
F^{\prime} \subseteq F, G^{\prime} \subseteq G, I^{\prime} \subseteq I, J^{\prime} \subseteq J, F \mathrm{R} G, F \cap I=\emptyset \text { and } G \cap J=\emptyset .
$$

Proof. Consider the following set of pairs of filters and ideals

$$
P=\left\{\left(F^{\prime \prime}, I^{\prime \prime}\right) \mid F^{\prime} \subseteq F^{\prime \prime}, I^{\prime} \subseteq I^{\prime \prime}, F^{\prime \prime} \cap I^{\prime \prime} \neq \emptyset \text { and } F^{\prime \prime} \mathrm{R} G^{\prime \prime}\right\} .
$$

If we take a chain of pairs $\left(F_{i}, I_{i}\right)$ from $P$, ordered by the condition $F_{i} \subseteq F_{j}$ and $I_{i} \subseteq I_{j}$ for $i<j$, then, by Lemma 1.3 (1.3), the chain has an upper bound in $P$. Then, by the Zorn's lemma, there is a maximal pair $(F, I)$. If $F \cup I \neq W$ then, by Lemma $1.5,(F, I)$ can be extended and is not maximal. So $F \cup I=W$ and, by Lemma 1.3(1.3), $F$ and $I$ are prime filter and ideal.

Because $\mathrm{R}$ is symmetric we can swap $F$ with $G^{\prime}$ and $I$ with $J^{\prime}$ and repeat the construction to obtain $G$ and $J$. Then swap again to achieve the final result.

In order to be able to use these lemmas, first, we have to prepare pairs of non-intersecting filters and ideals. Then we can expand them with either the Separation Lemma or the R-extension Lemma. We will illustrate the use of this procedure in the proof of the next lemma.

Lemma 1.7. Let $\underline{W}$ be a mereotopological structure and $x, y \in W$. Then

(1) if $x \not \leq y$ then there exists a prime filter (denoted by) $F(x \not \leq y)$, containing $x$ but not $y$;

(2) if $x \circ y$ then there exists a prime filter (denoted by $F(x \circ y)$ ) containing $x$ and $y$;

(3) if $x \cup y$ then there exists a prime filter (denoted by $F(x \cup y)$ ) containing neither $x$ nor $y$;

(4) if $x \mathrm{C} y$ then there exists a pair of prime filters $\langle F, G>$ such that $F \mathrm{R} G, x \in F$ and $y \in G$ (notation $<F, G>(x \subset y)$ ).

Proof. (1) We set $F^{\prime}=[x)$ and $I^{\prime}=(y]$. Since $x \not \leq y$, then by (M7) and (M11) $x \circ x$ and $y \cup y$. Then by Lemma $1.3(1)[x)$ is a filter 
and by Lemma $1.3(2)(y]$ is an ideal. Also, by Lemma 1.3 (?) we have $[x) \cap(y]=\emptyset$. Then we expand $F^{\prime}$ and $I^{\prime}$ with the Separation Lemma to $F$ and $I$, having $F \cap I=\emptyset$. Thus, $x \in F^{\prime} \subseteq F$ and since $y \in I^{\prime} \subseteq I$ then $y \notin F$. We take $F$ to be $F(x \not \leq y)$.

(2) We set $F^{\prime}=[x] \cup[y)$ and $I^{\prime}=\emptyset$ and proceed as in the previous case.

(3) Set $F^{\prime}=\emptyset$ and $I^{\prime}=(x] \cup(y]$.

(4) Set $F^{\prime}=[x), G^{\prime}=[y)$ and $I^{\prime}=J^{\prime}=\emptyset$ and use the R-extension Lemma to expand $F^{\prime}$ and $G^{\prime}$ to $F$ and $G$. This is the pair $\langle F, G\rangle$ $(x \subset y)$.

This lemma is actually half of the proof of the Static Characterization. Each of the statements there is an equivalence and one of the directions follows from the definition of prime filter of the definition of $\mathrm{R}$, while the other direction follows from Lemma 1.7.

\section{Dynamic mereological structures}

This section contains the preliminary results about the dynamic mereological theory, developed in [12]. It contains the definitions and the corresponding Stone-like representation theory for the dynamic variants of the relations $\leq, \mathrm{O}$ and $\mathrm{U}$. Since there are no changes from [12], then the proofs for the results from this section will be omitted here. For more more details see [12].

Definition 6. Let $I$ be a nonempty set of moments of time. For every $i \in I$, let $\underline{W}_{i}=\left(W_{i}, \leq_{i}, \mathrm{O}_{i}, \mathrm{U}_{i}\right)$ be a static mereological structure (see Definition 3). Let $W \subseteq \prod_{i \in I} W_{i}$, such that $W \neq \emptyset$. Then the stable and unstable mereological relations are defined for $x, y \in W$ as follows:

$$
\begin{array}{ccc}
x \leq y & \text { iff } & \forall i \in I, x_{i} \leq_{i} y_{i} \\
x \circ y & \text { iff } & \forall i \in I, x_{i} \mathrm{O}_{i} y_{i} \\
x \mathrm{u} y & \text { iff } & \forall i \in I, x_{i} \cup_{i} y_{i} \\
x \preceq y & \text { iff } & \exists i \in I, x_{i} \leq_{i} y_{i} \\
x \mathrm{O} y & \text { iff } & \exists i \in I, x_{i} \mathrm{O}_{i} y_{i} \\
x \cup y & \text { iff } & \exists i \in I, x_{i} \cup_{i} y_{i}
\end{array}
$$

$$
\begin{array}{r}
\text { stable part-of, } \\
\text { stable overlap, } \\
\text { stable underlap, } \\
\text { unstable part-of, } \\
\text { unstable overlap, } \\
\text { unstable underlap. }
\end{array}
$$

We call $\underline{W}=(W, \leq, \mathrm{o}, \mathrm{u}, \preceq, \mathrm{O}, \mathrm{U})$ a standard dynamic mereological structure. 
Notation. The complements of these six stable and unstable mereological relations will be denoted by $\npreceq, \overline{\mathrm{o}}, \overline{\mathrm{u}}, \npreceq, \overline{\mathrm{O}}$ and $\overline{\mathrm{U}}$.

Referring to the notations $R^{\forall}$ and $R^{\exists}$ from the introduction we have that

$$
\leq=\leq^{\forall}, \quad \mathrm{o}=\mathrm{O}^{\forall}, \quad \mathrm{u}=\mathrm{U}^{\forall}, \quad \preceq=\leq^{\exists}, \quad \mathrm{O}=\mathrm{O}^{\exists}, \quad \mathrm{U}=\mathrm{U}^{\exists}
$$

Note that the symbols for some of the relations (stable part-of and unstable overlap and underlap) are the same as the symbols for their static counterparts. This is so because the new dynamic structures satisfy the same conditions that the static mereological relations $\leq, \mathrm{O}$ and U do: (M1)-(M13).

Lemma 2.1. Let $\underline{W}$ be a standard dynamic mereological structure. Then it satisfies (M1)-(M13) and the following 20 new conditions

(M14) $x \preceq x$

(M15) $x \leq y \& y \preceq z \Longrightarrow x \preceq z$

(M16) $x \preceq y \& y \leq z \Longrightarrow x \preceq z$

(M17) $x \circ y \Longrightarrow y \circ x$

(M23) $x$ и $y \Longrightarrow y$ u $x$

(M18) $x \circ y \Longrightarrow x \circ x$

(M24) $x$ u $y \Longrightarrow x$ u $x$

(M19) $x \circ y \& y \leq z \Longrightarrow x \circ z$

(M25) $x \leq y \& y \mathrm{u} z \Longrightarrow x \mathrm{u} z$

(M20) $x \circ y \& y \preceq z \Longrightarrow x \mathrm{O} z$

(M26) $x \preceq y \& y \mathrm{u} z \Longrightarrow x \mathrm{U} z$

(M21) $x$ o $x$ or $x \preceq y$

(M28) $y$ u $y$ or $x \preceq y$

(M22) $x$ o $z$ or $y \mathrm{U} z$ or $x \preceq y$

(M27) $x \mathrm{O} z$ or $y \mathrm{u} z$ or $x \preceq y$

(M29) $x \circ x$ or $x \cup x$

(M30) $x$ O $x$ or $x \mathrm{u} x$

$(\mathrm{M} \leq) \quad x \leq y \Longrightarrow x \preceq y$

(Mo) $\quad x \circ y \Longrightarrow x \mathrm{O} y$

(Mu) $\quad x$ u $y \Longrightarrow x \mathrm{U} y$

$(\mathrm{M} \leq),(\mathrm{Mo})$ and $(\mathrm{Mu})$ are actually consequences of (M1)-(M30).

Thus, we give the general definition for the dynamic mereological structures

Definition 7. Let $\underline{W}=(W, \leq, \mathrm{o}, \mathrm{u}, \preceq, \mathrm{O}, \mathrm{U})$ be a relational structure, such that $W \neq \emptyset$. Then we call $\underline{W}$ a dynamic mereological structure if it satisfies the conditions (M1)-(M30). 


\subsection{Representation theory}

This theory is also a generalization of Stone's technique. Again we use the notion of prime filters to serve as abstract space points (see Definition 4). For the abstract time moments we use special sets of abstract points (they are called stable filter families in [12]). Each such set is, in fact, the set of all spatial points that exist at this moment of time.

Definition 8. Let $\underline{W}=(W, \leq, \mathrm{o}, \mathrm{u}, \preceq, \mathrm{O}, \mathrm{U})$ be a dynamic mereological structure. Let $\mathcal{F} \subseteq A P(\underline{W})$. We call $\mathcal{F}$ a mereological time moment iff $\mathcal{F} \neq \emptyset$ and $\mathcal{F}$ satisfies the following three conditions for all $x, y \in W$ :

(1) $x \npreceq y$ implies $\exists F \in \mathcal{F}, x \in F \& y \notin F$;

(2) $x$ ○ $y$ implies $\exists F \in \mathcal{F}, x \in F \& y \in F$;

(3) $x$ u $y$ implies $\exists F \in \mathcal{F}, x \notin F \& y \notin F$.

Notation. The set of all mereological time moments for $\underline{W}$ will be denoted by $M T M(\underline{W})$.

Note that all three conditions from this definition are, in fact, conditions for existence of specific abstract points. Thus, it is easy to see that if $\mathcal{F} \subseteq \mathcal{G} \subseteq A P(\underline{W})$ and $\mathcal{F}$ is a mereological time moment, then $\mathcal{G}$ is a mereological time moment too. Also, this allows us to construct a mereological time moment from any non-empty set of abstract points.

Lemma 2.2. Let $\mathcal{F}^{\prime}$ be a non-empty set of abstract points for a structure $\underline{W}$. Then there exists $\mathcal{F} \in M T M(\underline{W})$, such that $\mathcal{F}^{\prime} \subseteq \mathcal{F}$.

Proof. We start from $\mathcal{F}^{\prime}$ and add more abstract points ot it, in order to satisfy conditions (1), (2) and (3). For instance, for condition (1) if $x \npreceq y$ then this implies $x \not \leq y$ and we add the abstract point $F(x \not \leq y)$ from Lemma 1.7. Similarly, we add abstract points of types $F(x \bigcirc y)$ and $F(x \cup y)$ to satisfy conditions (2) and (3), respectively. Thus we set $\mathcal{F}$ to be

$$
\begin{aligned}
\mathcal{F}= & \mathcal{F}^{\prime} \cup\{F(x \not \leq y) \mid x, y \in W, x \npreceq y\} \cup \\
& \{F(x \circ y) \mid x, y \in W, x \circ y\} \cup\{F(x \cup y) \mid x, y \in W, x \text { u } y\} .
\end{aligned}
$$

Having the notions of abstract space point and abstract time moment we can characterize (represent) the dynamic mereological relations as follows 
Proposition 2.1 (Dynamic Mereological Characterization). For all dynamic mereological structures $\underline{W}=(W, \leq, \mathrm{o}, \mathrm{u}, \preceq, \mathrm{O}, \mathrm{U})$ and for all $x$, $y \in W:$

$(\leq) \quad x \leq y \quad$ iff $\quad \forall \mathcal{F} \in M T M(\underline{W}), \forall F \in \mathcal{F}, x \in F \Longrightarrow y \in F$;

(o) $\quad x \circ y \quad$ iff $\quad \forall \mathcal{F} \in M T M(\underline{W}), \exists F \in \mathcal{F}, x \in F \& y \in F$;

(u) $\quad x$ u $y$ iff $\quad \forall \mathcal{F} \in M T M(\underline{W}), \exists F \in \mathcal{F}, x \notin F \& y \notin F$;

$(\preceq) \quad x \preceq y \quad$ iff $\quad \exists \mathcal{F} \in M T M(\underline{W}), \forall F \in \mathcal{F}, x \in F \Longrightarrow y \in F$;

(O) $\quad x \mathrm{O} y \quad$ iff $\quad \exists \mathcal{F} \in M T M(\underline{W}), \exists F \in \mathcal{F}, x \in F \& y \in F$;

(U) $\quad x \cup y$ iff $\exists \mathcal{F} \in M T M(\underline{W}), \exists F \in \mathcal{F}, x \notin F \& y \notin F$.

Notice that the Dynamic Mereological Characterization is just a natural combination of the quantification over the moments of time $I$ from Definition 6 (but this time over the set of abstract moments of time $\operatorname{MTM}(\underline{W}))$ and the conditions from the Static Characterization.

\section{Dynamic mereotopological structures}

This section presents the new dynamic systems in this paper. We extend the dynamic mereological structures with stable and unstable variants of the contact relation and extend the set of first-order axioms with conditions for the two new relations. Here we use the following notations $\mathrm{C}=\mathrm{C}^{\forall}$ and $\mathrm{C}=\mathrm{C}^{\exists}$. Then we modify the representation theory in order to have characterizations for the new relations, as well.

Definition 9. Let $I$ be a nonempty set of moments of time and for all $i \in I$, let $\underline{W}_{i}=\left(W_{i}, \leq_{i}, \mathrm{O}_{i}, \mathrm{U}_{i}, \mathrm{C}_{i}\right)$ be a static mereotopological structure. Let $W \subseteq \prod_{i \in I} W_{i}$, such that $W \neq \emptyset$. Then we define $\mathrm{c}$ and $\mathrm{C}$ for all $x, y \in W$ as follows:

$$
\begin{aligned}
& x \subset y \quad \text { iff } \quad \forall i \in I, x_{i} \mathrm{C}_{i} y_{i} \quad \text { stable contact, } \\
& x \mathrm{C} y \quad \text { iff } \quad \exists i \in I, x_{i} \mathrm{C}_{i} y_{i} \quad \text { unstable contact. }
\end{aligned}
$$

Then the structure $\underline{W}=(W, \leq, \mathrm{o}, \mathrm{u}, \mathrm{c}, \preceq, \mathrm{O}, \mathrm{U}, \mathrm{C})$, where the relations $\leq$, $\mathrm{o}, \mathrm{u}, \preceq, \mathrm{O}$ and $\mathrm{U}$ are defined as in Definition 6, will be called a standard dynamic mereotopological structure or just standard structure for short.

Notation. The complements of the dynamic contacts will be denoted by $\overline{\mathrm{C}}$ and $\overline{\mathrm{C}}$. 
Lemma 3.1. Let $\underline{W}$ be a standard structure. Then it satisfies (M1)(M30), (C1)-(C4) and the following six conditions for the stable contact c:

(C5) $\quad x$ с $y \Longrightarrow y$ с $x$

(C7) $\quad x$ с $y \Longrightarrow x \circ x$

(C6) $x \circ y \Longrightarrow x \circ y$

(C8) $x \subset y \& y \leq z \Longrightarrow x \subset z$

(C9) $\quad x$ с $y \& y \preceq z \Longrightarrow x \mathrm{C} z$

$(\mathrm{C} 10) z \mathrm{c} t \& x \overline{\mathrm{u}} y \& z \overline{\mathrm{O}} y \& t \overline{\mathrm{O}} x \Longrightarrow x \mathrm{C} y$

Proof. The proof is just a straightforward check against Definition 9. We will show it only in the case of $(\mathrm{C} 10)$. The proof for the other conditions (M1)-(M30), (C1)-(C9) is similar.

Suppose that there is a standard structure $\underline{W}$, which does not satisfy (C10). This means that for $\underline{W}, z \mathrm{c} t, x \overline{\mathrm{u}} y, z \overline{\mathrm{O}} y, t \overline{\mathrm{O}} x$ and $x \overline{\mathrm{C}} y$ hold. Let $\underline{W}_{i}$ for $i \in I$ be the static structures from the definition of $\underline{W}$. Then, by Definition $9, z$ c $t$ means that for every $i \in I$ we have $z_{i} \mathrm{C}_{i} t_{i}$. Similarly, from $z \overline{\mathrm{O}} y, t \overline{\mathrm{O}} x$ and $x \overline{\mathrm{C}} y$ we get that for every $i \in I, z_{i} \overline{\mathrm{O}}_{i} y_{i}, t_{i} \overline{\mathrm{O}}_{i} x_{i}$ and $x_{i} \overline{\mathrm{C}}_{i} y_{i}$. Finally, from $x \overline{\mathrm{u}} y$ we have that there is $j \in I$ such that $x_{j} \overline{\mathrm{U}}_{j} y_{j}$. But then for that $j$ we have $z_{j} \mathrm{C}_{j} t_{j}$, $x_{j} \overline{\mathrm{U}}_{j} y_{j}, z_{j} \overline{\mathrm{O}}_{j} y_{j}, t_{j} \overline{\mathrm{O}}_{j} x_{j}$ and $x_{j} \overline{\mathrm{C}}_{j} y_{j}$. This is a contradiction with the condition from Lemma 1.2.

Similarly, if we suppose that a condition from (M1)-(M30), (C1)(C9) does not hold for $\underline{W}$, then we will get a contradiction that one of the static structures does not satisfy one of its defining conditions (M1)-(M13), (C1)-(C4) from Definition 3.

Lemma 3.1 suggests to give the following general definition of dynamic mereoto-pological structures

Definition 10. Let $\underline{W}=(W, \leq, \mathrm{o}, \mathrm{u}, \mathrm{c}, \preceq, \mathrm{O}, \mathrm{U}, \mathrm{C})$ be a relational structure, such that $W \neq \emptyset$. Then $\underline{W}$ is called a dynamic mereotopological structure or just an dynamic structure iff it satisfies (M1)-(M30) and (C1) $-(\mathrm{C} 10)$.

\subsection{Representation theory}

We aim to show that every dynamic structure can be represented as (isomorphic to) a standard one. For this purpose we use the notion of prime filters from Definition 4 again. We use them to recreate the space points. To recreate the moments of time, however, we will have 
to change the notion of mereological time moments (Definition 8). The reason is the addition of the contact relations $\mathrm{c}$ and $\mathrm{C}$ to the system. The mereological time moments, being sets of abstract space points, can generate Boolean algebras and the mereological relations can be defined (represented) in Boolean algebras. But to define (represent) the contacts we need contact algebras. One possible way to obtain contact algebras is through reflexive and symmetric relational systems (Definition 2). Thus, we add to the set of space points a reflexive and symmetric relation, based on the relation $\mathrm{R}$ (from Definition 5).

Definition 11. Let $\underline{W}=(W, \leq, \mathrm{o}, \mathrm{u}, \mathrm{c}, \preceq, \mathrm{O}, \mathrm{U}, \mathrm{C})$ be a dynamic structure. Let $\mathcal{F}$ be a mereological time moment for $\underline{W}$ and $\mathcal{R}$ is a reflexive and symmetric binary relations over $\mathcal{F}$. Then the relational system $(\mathcal{F}, \mathcal{R})$ is called an abstract time moment (or just an abstract moment) if for all $x, y \in W, x \mathcal{R} y$ implies $x \mathrm{R} y$ (i.e. $\mathcal{R}$ is a subrelation of $\mathrm{R}$ from Definition 5) and this condition holds for all $x, y \in W$ :

(4) $x$ с $y$ implies $\exists F, G \in \mathcal{F}, x \in F \& y \in G \& F \mathcal{R} G$.

Notation. The set of all abstract moments for $\underline{W}$ will be denoted by $A M(\underline{W})$.

Every mereological time moment can be extended to an abstract time moment.

Lemma 3.2. Let $\mathcal{F}^{\prime}$ be a mereological time moment for a dynamic structure $\underline{W}$. Then there exists $\mathcal{F} \in A M(\underline{W})$, such that $\mathcal{F}^{\prime} \subseteq \mathcal{F}$.

Proof. To satisfy condition (4), extend $\mathcal{F}^{\prime}$ by adding a pair of abstract points for each $x$ and $y$ such that $x \subset y$. Since $x \subset y$ implies $x \mathrm{C} y$, the pair of abstract points is $\langle F, G\rangle(x \subset y)$ from Lemma 1.7.

$$
\mathcal{F}=\mathcal{F}^{\prime} \cup\{H \mid x, y \in W, x \subset y, H \in\langle F, G\rangle(x \subset y)\}
$$

Finally, take $\mathcal{R}$ to be the restriction of $\mathrm{R}$ from Definition 5 to $\mathcal{F}$, i.e. $\mathcal{R}=\mathrm{R}_{\mid \mathcal{F}}$.

Similarly to the previous section, this allows us to extend any nonempty set of abstract points to an abstract time moment. First, extend the set to a mereological time moment, by Lemma 2.2. Then extend the result to an abstract time moment, by the above Lemma 3.2. 
Thus, the new characterization of the dynamic relations is

Proposition 3.1 (Dynamic Mereotopological Characterization). For all dynamic structures $\underline{W}=(W, \leq, \mathrm{o}, \mathrm{u}, \mathrm{c}, \preceq, \mathrm{O}, \mathrm{U}, \mathrm{C})$ and for all $x, y \in W$ :

$(\leq) x \leq y$ iff $\forall(\mathcal{F}, \mathcal{R}) \in A M(\underline{W}), \forall F \in \mathcal{F}, x \in F \Longrightarrow y \in F$;

(o) $x$ ○ $y$ iff $\forall(\mathcal{F}, \mathcal{R}) \in A M(\underline{W}), \exists F \in \mathcal{F}, x \in F \& y \in F$;

(u) $\quad x$ u $y$ iff $\forall(\mathcal{F}, \mathcal{R}) \in A M(\underline{W}), \exists F \in \mathcal{F}, x \notin F \& y \notin F$;

(c) $x$ c $y$ iff $\forall(\mathcal{F}, \mathcal{R}) \in A M(\underline{W}), \exists F, G \in \mathcal{F}, x \in F \& y \in G \& F \mathcal{R} G$;

$(\preceq) x \preceq y$ iff $\exists(\mathcal{F}, \mathcal{R}) \in A M(\underline{W}), \forall F \in \mathcal{F}, x \in F \Longrightarrow y \in F$;

(O) $x$ O $y$ iff $\exists(\mathcal{F}, \mathcal{R}) \in A M(\underline{W}), \exists F \in \mathcal{F}, x \in F \& y \in F$;

(U) $x \mathrm{U} y$ iff $\exists(\mathcal{F}, \mathcal{R}) \in A M(\underline{W}), \exists F \in \mathcal{F}, x \notin F \& y \notin F$;

(C) $x \subset y$ iff $\exists(\mathcal{F}, \mathcal{R}) \in A M(\underline{W}), \exists F, G \in \mathcal{F}, x \in F \& y \in G \& F \mathcal{R} G$.

Proof. See Appendix A.

Finally we apply the Dynamic Mereotopological Characterization in the proof of the central representation theorem.

Theorem 3.1 (Representation Theorem). Let $\underline{W}^{d}=\left(W^{d}, \leq^{d}, \mathrm{o}^{d}, \mathrm{u}^{d}, \mathrm{c}^{d}, \preceq^{d}, \mathrm{O}^{d}, \mathrm{U}^{d}, \mathrm{C}^{d}\right)$ be a dynamic structure. Then there exists a standard structure $\underline{W}$ isomorphic to $\underline{W}^{d}$.

PROOF. The proof is very similar to that of the main representation theorem in [12]. First we will prove that there exists a standard structure $\underline{W^{\prime}}=\left(W^{\prime}, \leq^{\prime}, \mathrm{o}^{\prime}, \mathrm{u}^{\prime}, \mathrm{c}^{\prime}, \preceq^{\prime}, \mathrm{O}^{\prime}, \mathrm{U}^{\prime}, \mathrm{C}^{\prime}\right)$ and an isomorphic embedding $h$ from $\underline{W}^{d}$ into $\underline{W}^{\prime}$. Let $I=A M\left(\underline{W}^{d}\right)$. Then let for every $i \in I, \underline{W}_{i}$ be the full static mereotopological structure defined by $i$ (see Definition 2, here $i$ is a reflexive and symmetric relational system). Then we define $\underline{W}^{\prime}$ by the structures $\underline{W}_{i}$ as in Definition 9. The embedding $h$ is defined for every $x \in W^{d}$ as we set each coordinate of the vector $h(x)$ :

$$
(h(x))_{i}=\{F \mid i=(\mathcal{F}, \mathcal{R}), F \in \mathcal{F}, x \in F\} .
$$

The proof that $h$ is injective and preserves the relations is the same as in [12]: use the Dynamic Mereotopological Characterization for $\underline{W}^{d}$ and the standard definition of $\underline{W^{\prime}}$ (Definition 9).

The injectiveness of $h$ is proved as follows: let $x, y \in W^{A}$, such that $x \neq y$; then, by (M3), $x \not \leq y$ or $y \not \leq x$; if $x \not \leq y$ then from the characterization of $\leq$ we have that there is an abstract moment $(\mathcal{F}, \mathcal{R})$ 
and $F \in \mathcal{F}$ such that $x \in F$ and $y \notin F$; this leads to the fact that $h(x)$ and $h(y)$ differ in their $i^{\text {th }}$ coordinate, where $i=(\mathcal{F}, \mathcal{R})$, because $F \in(h(x))_{i}$ but $F \notin(h(y))_{i}$; thus, $h(x) \neq h(y)$; the case $y \not \leq x$ is checked in the same way.

Here is an example proof that $h$ preserves one of the new relations. The proofs for the rest of the relations are similar.

$$
\begin{array}{rll}
x c^{d} y \quad \text { iff } & \forall(\mathcal{F}, \mathcal{R}) \in A M\left(\underline{\underline{W}}^{d}\right), \exists F, G \in \mathcal{F}, x \in F \& y \in G \& F \mathcal{R} G \\
\text { iff } & \forall i \in I, \exists F \in(h(x))_{i}, \exists G \in(h(y))_{i}, F \mathcal{R} G \\
\text { iff } & \forall i \in I,(h(x))_{i} \mathrm{C}_{i}(h(y))_{i} \quad \text { iff } \quad h(x) c^{\prime} h(y)
\end{array}
$$

Finally, let $\underline{W}$ be the substructure of $\underline{W^{\prime}}$ such that the universe of $\underline{W}$ is exactly range $(h)$. Thus, $h$ is an isomorphic embedding from $\underline{W}^{d}$ to $\underline{W}$, as well, and now $h$ is a bijection. So $\underline{W}$ is isomorphic to $\underline{W}^{d}$.

\section{First-order logics of dynamic mereotopological relations}

In this section we study the first-order logics of dynamic mereotopological relations, their completeness and decidability. Since the full firstorder logic turns out to be undecidable, it makes sense to consider its decidable quantifier-free fragment and the complexity of the fragment.

\subsection{The full first-order logic}

The language of this logic is a first-order language with no functional symbols, no constants and the predicate symbols being the eight dynamic mereotopological relations and equality. The standard structures will serve as models for the logic. The models will be denoted by $(\underline{W}, v)$ where $\underline{W}$ is the standard structure and $v$ is valuation of the variables into the universe of the structure.

The completeness of the logic is proved with the help of the Representation Theorem. We will also show that this logic is hereditary undecidable. This means that its minimal theory is undecidable and any of its sub-theories in the same language is also undecidable.

\section{Completeness}

We use the standard first-order axiom system for the language of the logic and extend it with the universal closures of conditions (M1)-(M30), 
(C1)-(C10). We will prove that the logic is complete with respect to this axiomatization.

Theorem 4.1. The first-order logics of dynamic mereotopological relations is complete with respect to (M1)-(M30), (C1)-(C10).

Proof. Let $\alpha$ be a formula of the logic, which is not a theorem. We will show that there is a standard structure in which $\alpha$ is not true.

By the Lindenbaum's lemma there is a maximal consistent set $\Gamma$, that does not contain $\alpha$. Lets consider the canonical structure $\underline{W}^{\prime}$ for $\Gamma$, produced by the Henkin construction. Thus, $\alpha$ is not true in $\underline{W^{\prime}}$. Since (M1)-(M30), (C1)-(C10) are axioms of the logic, then all of them are included in $\Gamma$ and are true in $\underline{W}^{\prime}$. So, by Definition $10, \underline{W^{\prime}}$ is a dynamic structure. Then, by the Representation Theorem, there is a standard structure $\underline{W}$ which is isomorphic to $\underline{W^{\prime}}$ and thus $\alpha$ is not true in $\underline{W}$.

\section{Hereditary undecidability}

The hereditary undecidability of the logic follows from a result in [13]. The result shows that every logic without functional symbols and constants, which includes the overlap relation or the contact relation in its language, is hereditary undecidable. The proof goes through use of a method described in [7]. Hereditary undecidability is shown by proving that the logic in question is relatively elementary interpretable in another hereditary undecidable logic. In this case we use the theory of the class of finite structures with a single symmetric and irreflexive relation. For details see [13].

\subsection{The quantifier-free fragment}

This is the logic of dynamic mereotopological relations without use of quantifiers. The language of this fragment consists of object variables, predicate symbols for every one of the eight dynamic relations, predicate for equality and the propositional connectives. Again we use standard structures as models for the logic.

First we will show that this fragment has a complete axiomatization by adding axioms (M1)-(M30), (C1)-(C10) to the set of Boolean tautologies. We will also show that it is decidable and its satisfiability problem is an NP-complete problem. 


\section{Completeness}

The axiomatization of the fragment is made of the Boolean tautologies, axioms (M1)-(M30), (C1)-(C10) and some of the equality axioms. In fact the axioms describing $=$ as an equivalence relation and

$(\mathrm{M}=)$

$$
x=y \Longrightarrow x \leq y
$$

are sufficient. The equality axioms for the other predicate symbols can be obtained as consequences of $(\mathrm{M}=)$ and other axioms amongst (M1)(M30), (C1)-(C10). The completeness is proved via a canonical model construction which is a simplified version of the Henkin canonical construction for the first-order logic. For more details see [12], where the same construction is described for the first-order logic of dynamic mereological relations. Also, proofs for similar systems can be found in [2] as well as in [23].

\section{Decidability and NP-completeness}

We will show that the fragment is decidable and its satisfiability problem is NP-complete. The same complexity is proved for very similar temporalized spatial logic in [10]. There the box and diamond operators from LTL are applied to formulae from the language RC for reasoning about regions from topological spaces. This in some sense corresponds to our stable and unstable variants of the base mereological relations. In our case we can say that the S5 box and diamond operators are applied to the atomic mereological formulae. Here we will prove the decidability and the complexity of this fragment by showing that it has the polysize model property.

Proposition 4.1. The quantifier-free fragment of the first-order logic of dynamic mereotopological relations possesses the polysize model property.

Proof. Let $\alpha$ be a quantifier-free formula and let $(\underline{W}, v)$ be a model such that $\underline{W}$ is a standard structure and $v$ is a valuation of the object variables. We will build a finite model $\left(\underline{W^{\prime}}, v^{\prime}\right)$ that preserves the truth of the formula. Let $\operatorname{Var}(\alpha)$ denote the set of all object variables in $\alpha$. Then let $\underline{W^{\prime}}$ be the substructure of $\underline{W}$ with universe $\{v(x) \mid x \in \operatorname{Var}(\alpha)\}$. Thus the size of $\underline{W^{\prime}}$ is limited by the size of $\alpha$. Then let $v^{\prime}$ be just an arbitrary valuation in $W^{\prime}$ such that $v^{\prime}(x)=v(x)$ for all $x \in \operatorname{Var}(\alpha)$. 
Since there are no quantifiers in the language, $\alpha$ is just a boolean combination of atomic formulae of the kind $x R y$. Then its truth depends only on the valuation of the variables from $\operatorname{Var}(\alpha)$. Thus, $\alpha$ is true in $(\underline{W}, v)$ iff $\alpha$ is true in $\left(\underline{W^{\prime}}, v^{\prime}\right)$.

Thus, we may conclude that

TheOrem 4.2. The quantifier-free fragment of the first-order logic of $d y$ namic mereotopological relations is decidable and its satisfiability problem is NP-complete

\section{Concluding remarks}

In conclusion we list some of the open problems and possible future developments of the topic presented in this paper.

The first line of development is to add more relations to the base set of four mereotopological relations. Here we aim to add as many such relations as possible. For example, we may add more relations from the RCC- 8 system or other relations of topological nature. However, the more relations that we add, the more complex constructions for the representation theory have to be used. For instance, in [12] to characterize stable and unstable variants of three mereological relations, nine auxiliary lemmas (similar to lemmas A.1 to A.4) for construction of specific prime filters were needed. Here, to characterize stable and unstable variants of four mereotopological relations, we use four new lemmas (A.1 to A.4) in addition to these nine.

The structure of time in the dynamic structures can also be a subject of further study. The use of some ordering of the time may introduce a whole new array of dynamic versions of relations - not only stable (always) and unstable (sometimes), but before, until, since, etc. This will have great effect on the representation theory and may force us to use different techniques for axiomatizing the relations (maybe standard methods from temporal logics, rather than from mereotopology and contact algebras).

In the end there is one more open issue: the decidability problem for the modal logic for stable and unstable mereotopological relations and its complexity (if it is decidable indeed). Like in [12] we may develop a poly-modal logic with universal modality with Kripke semantics over the dynamic structures. Its completeness is proved in the same way as in [12]: use a variation of Segerberg's bulldozer construction to replace the 
modally undefinable condition (M3) with modally definable conditions; then the completeness is easily proven by means of generated canonical models. The conjecture is that the modal logic possesses the strong form of the finite model property and that this can be proved with the filtration technique with similar filtration conditions to those from [12].

\section{A. Proof of the Dynamic Mereotopological Characterization (Proposition 3.1)}

The proof will make use of lemmas A.1, A.2, A.3 and A.4 (see below).

Proof of Proposition 3.1. We have to prove that for every dynamic structure $\underline{W}$ and for all $x, y \in W$

( $\leq) \quad x \leq y$ iff $\forall(\mathcal{F}, \mathcal{R}) \in A M(\underline{W}), \forall F \in \mathcal{F}, x \in F \Longrightarrow y \in F$;

(o) $x \circ y$ iff $\forall(\mathcal{F}, \mathcal{R}) \in A M(\underline{W}), \exists F \in \mathcal{F}, x \in F \& y \in F$;

(u) $\quad x$ u $y$ iff $\forall(\mathcal{F}, \mathcal{R}) \in A M(\underline{W}), \exists F \in \mathcal{F}, x \notin F \& y \notin F$;

(c) $x$ c $y$ iff $\forall(\mathcal{F}, \mathcal{R}) \in A M(\underline{W}), \exists F, G \in \mathcal{F}, x \in F \& y \in G \& F \mathcal{R} G$;

$(\preceq) x \preceq y$ iff $\exists(\mathcal{F}, \mathcal{R}) \in A M(\underline{W}), \forall F \in \mathcal{F}, x \in F \Longrightarrow y \in F$;

(O) $x$ O $y$ iff $\exists(\mathcal{F}, \mathcal{R}) \in A M(\underline{W}), \exists F \in \mathcal{F}, x \in F \& y \in F$;

(U) $x \cup y$ iff $\exists(\mathcal{F}, \mathcal{R}) \in A M(\underline{W}), \exists F \in \mathcal{F}, x \notin F \& y \notin F$;

(C) $x$ C $y$ iff $\exists(\mathcal{F}, \mathcal{R}) \in A M(\underline{W}), \exists F, G \in \mathcal{F}, x \in F \& y \in G \& F \mathcal{R} G$.

First we show the proof of condition $(\leq)$.

$(\longrightarrow)$ If $x \leq y$ and $(\mathcal{F}, \mathcal{R})$ is an abstract moment and $F \in \mathcal{F}$ then, because $F$ is a abstract point, the Static Characterization shows that $x \in F$ implies $y \in F$.

$(\longleftarrow)$ Suppose that the right-hand side of the condition holds but $x \not \leq y$. We will show that $x \not \leq y$ implies that there is an abstract moment, that is a counter-example for the right-hand side condition and thus leading to contradiction. By the Dynamic Mereological Characterization $x \not \leq y$ implies that there is a mereological time moment $\mathcal{F}^{\prime}$ containing an abstract point $F$ such that $x \in F$ and $y \notin F$. Then we obtain $(\mathcal{F}, \mathcal{R})$ from $\mathcal{F}^{\prime}$, as in Lemma 3.2. Thus, $(\mathcal{F}, \mathcal{R})$ is the needed abstract moment that serves as counter-example.

The conditions for $\mathrm{O}$ and $\mathrm{U}$ are proved similarly to $\leq$. Also, the conditions for $\circ$ and $\preceq$ are proved in the same way as u. Lemmas A.2 
and A.3 should be used in the cases of o and $\preceq$, instead of Lemma A.1. We show the proof only for $u$.

$(\longrightarrow)$ If $x$ u $y$ and $(\mathcal{F}, \mathcal{R})$ is an abstract moment, by Definition 8 (3), there is $F \in \mathcal{F}$ such that $x \notin F$ and $y \notin F$.

$(\longleftarrow)$ We will prove that if $x \overline{\mathrm{u}} y$ holds, then there is an abstract moment, which is a counter-example for the right-hand side condition. By the Dynamic Mereological Characterization, $x \overline{\mathrm{u}} y$ implies the existence of a mereological time moment $\mathcal{F}^{\prime}$, such that for every $F \in \mathcal{F}^{\prime}, x \in F$ or $y \in F$. Then, as in Lemma 3.2, we obtain $(\mathcal{F}, \mathcal{R})$ as we extend $\mathcal{F}^{\prime}$ to $\mathcal{F}$, this time with $\{H \mid z, t \in W, z \mathrm{c} t, H \in\langle F, G\rangle(x \overline{\mathrm{u}} y, z \mathrm{c} t)\}$ (the pairs $\langle F, G\rangle(x \overline{\mathrm{u}} y, z \mathrm{c} t)$ are defined with Lemma A.1), and again take $\mathcal{R}=\mathrm{R}_{\mid \mathcal{F}}$. By Lemma A.1, we have that for every of the new abstract points, added to $\mathcal{F}, x \in H$ or $y \in H$. Thus $(\mathcal{F}, \mathcal{R})$ is the needed abstract moment.

Here follows the proof for the condition for c.

$(\longrightarrow)$ This direction follows from Definition 11(4).

$(\longleftarrow)$ We will prove that $x \overline{\mathrm{c}} y$ implies that there is a counter-example of the right-hand side. $x \bar{c} y$ implies $x \bar{o} y$, by (C6). Thus, by the Dynamic Mereological Characterization, there is a mereological time moment $\mathcal{F}^{\prime}$ such that for every $F \in \mathcal{F}^{\prime}, x \notin F$ or $y \notin F$. Then take $\mathcal{F}=\mathcal{F}^{\prime} \cup\{H \mid z, t \in W, z \mathrm{c} t, H \in\langle F, G\rangle(x \overline{\mathrm{c}} y, z \mathrm{c} t)\}$ and $\mathcal{R}=\mathrm{R}_{\mid \mathcal{F}} \backslash\{\langle F, G\rangle,\langle G, F\rangle \mid x \in F, y \in G, F \mathrm{R} G\}$. In essence, we remove the pairs from $\mathrm{R}_{\mid \mathcal{F}}$ that would satisfy the the right-hand side condition. Thus, if $(\mathcal{F}, \mathcal{R})$ is an abstract moment it will be a counterexample. By construction $\mathcal{R}$ is symmetric. By Lemma A.4, for every $H \in\langle F, G\rangle(x \overline{\mathrm{c}} y, z \mathrm{c} t)$ we have $x \notin H$ or $y \notin H$ and so this is true for every abstract point in $\mathcal{F}$. Thus all pairs $\langle F, F\rangle$ remain in $\mathcal{R}$ and it is reflexive. Finally, $(\mathcal{F}, \mathcal{R})$ satisfies condition (4) from Definition 11, because, by Lemma A.4, the pairs $\langle F, G\rangle(x \overline{\mathrm{c}} y, z \mathrm{c} t)$ do not satisfy $x \in F, y \in G$ and $F \mathrm{R} G$ and so they will not be removed from $\mathcal{R}$.

Finally we prove the condition for $\mathrm{C}$.

$(\longrightarrow)$ By the Static Characterization, $x$ C $y$ implies that there are abstract points $F$ and $G$ so that $x \in F, y \in G$ and $F$ R $G$. Then expand the set $\{F, G\}$ to the mereological time moment $\mathcal{F}^{\prime}$ as in Lemma 2.2. Then obtain the needed abstract moment $\left(\mathcal{F}, \mathcal{R}\right.$ ) from $\mathcal{F}^{\prime}$ (see Lemma 3.2). $(\longleftarrow) F \mathcal{R} G$ implies $F \mathrm{R} G$. So $x \subset y$ follows from the definition of R. 
Lemma A.1 (Abstract point for $x \overline{\mathrm{u}} y$ and $z \mathrm{c} t$ ). Let $\underline{W}$ be any dynamic mereotopological structure and $x, y, z, t \in W$ such that $x \overline{\mathrm{u}} y$ and $z \mathrm{c} t$. Then there is a pair of prime filters $F$ and $G$ such that $z \in F, t \in G$, $F \mathrm{R} G$ and both $F$ and $G$ contain at least one of $x$ and $y$.

Notation. Such pair will be denoted by $\langle F, G\rangle(x \overline{\mathrm{u}} y, z \mathrm{c} t)$.

Proof. Let $I^{\prime}=J^{\prime}=\emptyset$. They are ideals. We will show that we can set $F^{\prime}$ to be either $F^{\prime}=[z) \cup[x)$ or $F^{\prime}=[z] \cup[y)$ and set either $G^{\prime}=[t] \cup[x)$ or $G^{\prime}=[t] \cup[y)$ and in every case to have that $F^{\prime}$ and $G^{\prime}$ are filters such that $F^{\prime} \mathrm{R} G^{\prime}$. Then we have that $z \in F^{\prime}, t \in G^{\prime}$ and both $F^{\prime}$ and $G^{\prime}$ contain at least one of $x$ and $y$. Since $F^{\prime} \cap I^{\prime}=\emptyset$ and $G^{\prime} \cap J^{\prime}=\emptyset$ then we have all required premises to apply the R-extension Lemma for $F^{\prime}$, $G^{\prime}, I^{\prime}$ and $J^{\prime}$ and obtain the needed prime filters $F$ and $G$.

Now consider several possible cases

1) $z O x$ and $t O x$. Then, by Lemma $1.3(1), F^{\prime}=[z) \cup[x)$ and $G^{\prime}=[t) \cup[x)$ are filters. By (C2), $z \mathrm{O} x$ and $t \mathrm{O} x$ imply $z \mathrm{C} x$ and $t$ C $x . z$ c $t$ implies $z$ C $t$. By (M4), (M5) and (C2), $z \mathrm{O} x$ implies $x \mathrm{C} x$. Thus, by Lemma 1.3(3), we have $[z) \mathrm{R}[t],[z] \mathrm{R}[x),[t] \mathrm{R}[x]$ and $[x) \mathrm{R}[x)$ and so $F^{\prime} \mathrm{R} G^{\prime}$.

2) $z O y$ and $t \bigcirc y$. This case is similar. Set $F^{\prime}=[z] \cup[y)$ and $G^{\prime}=[t) \cup[y)$.

If the first two cases are not true then we have that $z \overline{\mathrm{O}} x$ or $t \overline{\mathrm{O}} x$ and also $z \overline{\mathrm{O}} y$ or $t \overline{\mathrm{O}} y$. These are four cases. We will show, however, that $z \overline{\mathrm{O}} x$ implies $z \mathrm{O} y$ and $t \overline{\mathrm{O}} y$ implies $t \mathrm{O} x$. Thus the possible cases are really two - case 3) in which $z \overline{\mathrm{O}} x$ and $t \overline{\mathrm{O}} y$ and case 4) for $z \overline{\mathrm{O}} y$ and $t \overline{\mathrm{O}} x$.

By (C3), $z$ c $t$ implies $z$ o $z$. By (M20), $z$ o $z$ and $z \overline{\mathrm{O}} x$ imply $z \npreceq x$. Then, by (M27), $z \npreceq x$ and $x \overline{\mathrm{u}} y$ imply $z \mathrm{O} y$. Similarly, $t \overline{\mathrm{O}} y$ implies $t O x$.

3) $z \overline{\mathrm{O}} x$ and $t \overline{\mathrm{O}} y$. They imply $z \mathrm{O} y$ and $t \mathrm{O} x$. Thus take $F^{\prime}=[z) \cup[y)$ and $G^{\prime}=[t) \cup[x) . z$ c $t$ implies $z \mathrm{C} t$. By (M27), $z \overline{\mathrm{O}} x$ and $x \overline{\mathrm{u}} y$ imply $z \preceq y$. Similarly we have $t \preceq x$. Combine these results with $z \mathrm{c} t$ and, by (C9), we get $z \mathrm{C} x$ and $t \mathrm{C} y$. Thus, by Lemma 1.3 (3), we have $[z] \mathrm{R}[t],[z] \mathrm{R}[x]$ and $(t) \mathrm{R}(y)$. From $z \mathrm{c} t, x \overline{\mathrm{u}} y, z \overline{\mathrm{O}} x$ and $t \overline{\mathrm{O}} y$, by (C10), we get $x \mathrm{C} y$. So $[x) \mathrm{R}[y)$ and finally $F^{\prime} \mathrm{R} G^{\prime}$.

4) $z \overline{\mathrm{O}} y$ and $t \overline{\mathrm{O}} x$. This case is proved the same way as the previous one. 
In the proofs of the next three lemmas we will only build the initial filters and ideals $F^{\prime}, G^{\prime}, I^{\prime}$ and $J^{\prime}$. In every case the proof can be finished by applying the R-extension Lemma to get $F$ and $G$.

Lemma A.2 (Abstract point for $x \overline{\mathrm{o}} y$ and $z \mathrm{c} t$ ). Let $\underline{W}$ be any dynamic mereotopological structure and $x, y, z, t \in W$ such that $x \overline{\mathrm{o}} y$ and $z \mathrm{c} t$. Then there is a pair of prime filters $F$ and $G$ such that $z \in F, t \in G$, $F \mathrm{R} G$ and both $F$ and $G$ do not contain at least one of $x$ and $y$.

Proof. From $z \mathrm{c} t$, by (C7), it follows that $z$ o $z$ and, by (Mo), we have $z \mathrm{O} z$. So, by Lemma 1.3(1), $F^{\prime}=[z)$ is a filter. Now consider the two possible cases $-z \not \leq x$ or $z \leq x$.

1) $z \not \leq x$. By (M11), also $x \mathrm{U} x$. So, by Lemma $1.3(2), I^{\prime}=(x]$ is an ideal and, because $z \not \leq x$ and by Lemma $1.3\left(\right.$ ?), $F^{\prime} \cap I^{\prime}=\emptyset$.

2) $z \leq x$. If $z \leq y$ then from $z \circ z$, by applications of (C8) and (C5), we get $x \subset y$. So $z \not \leq y$ and thus $y \mathrm{U} y$ and $I^{\prime}=(y]$ is an ideal and $F^{\prime} \cap I^{\prime}=\emptyset$.

Thus in either case we have an ideal $I^{\prime}$ such that $F^{\prime} \cap I^{\prime}=\emptyset$ and $x \in I^{\prime}$ or $y \in I^{\prime}$ (this means that $x \notin F^{\prime}$ or $y \notin F^{\prime}$ ). Similarly we get a filter $G^{\prime}=[t)$ and an ideal $J^{\prime}$ such that $G^{\prime} \cap J^{\prime}=\emptyset$ and $x \in J^{\prime}$ or $y \in J^{\prime}$. Since $z$ c $t$, by Lemma $1.3(3)$, then $F^{\prime} \mathrm{R} G^{\prime}$.

Lemma A.3 (Abstract point for $x \preceq y$ and $z \mathrm{c} t$ ). Let $\underline{W}$ be any dynamic mereotopological structure and $x, y, z, t \in W$ such that $x \preceq y$ and $z \mathrm{c} t$. Then there is a pair of prime filters $F$ and $G$ such that $z \in F, t \in G$, $F \mathrm{R} G$ and both $F$ and $G$ satisfy that if they contain $x$ then they contain $y$.

Proof. From $z \mathrm{c} t$, by $(\mathrm{C} 7)$ and (C5), we have $z \circ z$ and $t \circ t$ and, by (Mo), $z \mathrm{O} z$ and $t \mathrm{O} t$. Consider the four cases about $z \leq x$ and $t \leq x$ :

$1) z \not \leq x$ and $t \not \leq x$. So $[z) \cap(x]=\emptyset$ and $[t) \cap(x]=\emptyset$. By (M11), $x \mathrm{U} x$ and we take $F^{\prime}=[z), G^{\prime}=[t)$ and $I^{\prime}=J^{\prime}=(x]$.

2) $z \not \leq x$ and $t \leq x . z \not \leq x$, by (M11), implies $x \cup x$ and take $F^{\prime}=[z)$ and $I^{\prime}=(x]$. From $t \leq x$ and $x \preceq y$, by (M15), $t \preceq y$. Then add $t \circ t$ and, by (M20), we get $t \bigcirc y$. Thus take $G^{\prime}=[t) \cup[y)$ and $J^{\prime}=\emptyset$. Finally, from $z \mathrm{c} t$ and $t \mathrm{O} y$, by (C9), we have $z \mathrm{C} y$ and thus $F^{\prime} \mathrm{R}[y)$. Because $z \mathrm{c} t$ implies $z \mathrm{C} t$, then $F^{\prime} \mathrm{R}[t)$. So $F^{\prime} \mathrm{R} G^{\prime}$.

3) $z \leq x$ and $t \not \leq x$. This case is the same as the previous one.

4) $z \leq x$ and $t \leq x$. Similarly to the previous two cases we get $z \mathrm{O} y$, $t \mathrm{O} y, z \mathrm{C} y$ and $t \mathrm{C} y$. From $z \mathrm{O} y$, by (M4), (M5) and (C2), we have $y$ C $y$. Thus $F^{\prime}=[z) \cup[y), G^{\prime}=[t] \cup[y)$ and $I^{\prime}=J^{\prime}=\emptyset$. 
In every case we have $x \notin F^{\prime}$ or $y \in F^{\prime}$ which means that $x \in F^{\prime}$ implies $y \in F^{\prime}$. The same goes for $G^{\prime}$.

Lemma A.4 (Abstract point for $x \overline{\mathrm{c}} y$ and $z \mathrm{c} t$ ). Let $\underline{W}$ be any dynamic mereotopological structure and $x, y, z, t \in W$ such that $x \overline{\mathrm{c}} y$ and $z \mathrm{c} t$. Then there is a pair of prime filters $F$ and $G$ such that $z \in F, t \in G$, $F \mathrm{R} G$, it is true that $x \notin F$ or $y \notin G$ and also $x \notin G$ or $y \notin F$.

Notation. Such pair will be denoted by $\langle F, G\rangle(x \bar{c} y, z \mathrm{c} t)$.

Proof. Suppose $z \leq x$ and $t \leq y$. Then from $z \mathrm{c} t$, by applications of (C8) and (C5), we get $x$ c $y$. It is the same if $z \leq y$ and $t \leq x$. So $z \not \leq x$ or $t \not \leq y$ and also $z \not \leq y$ or $t \not \leq x$. Thus we have four cases:

$1) z \not \leq x$ and $t \not \leq x$. By (M11), $z \not \leq x$ implies $x \cup x$. By (C7) and (C5), $z$ c $t$ implies $z \circ z$ and $t \circ t$ and, by (Mo), we have $z \mathrm{O} z$ and $t \mathrm{O} t$. Thus take $F^{\prime}=[z), G^{\prime}=[t)$ and $I^{\prime}=J^{\prime}=(x]$.

2) $t \not \leq y$ and $z \not \leq y$. This case is the same as the previous one.

$3) z \not \leq x$ and $z \not \leq y$. By (C5), (C7) and (C6), $z$ c $t$ implies $t$ c $t$. If $t \leq x$ and $t \leq y$ then, by (C8) and (C5), we get $x$ c $y$. So $t \not \leq x$ or $t \not \leq y$ and thus we reduce this case to the first two cases.

4) $t \not \leq y$ and $t \not \leq x$. This case is the same as the previous one.

Acknowledgments. Thanks are due to an anonymous referee for the very helpful remarks that aided in improving the quality of this paper. The work of the author is supported by the project DID02/32/2009 of Bulgarian NSF.

\section{References}

[1] Balbes, R., and P. Dwinger, Distributive Lattices, University of Missouri Press, 1974.

[2] Balbiani, P., T. Tinchev, and D. Vakarelov, "Modal logics for region-based theory of space", Fundamenta Informaticae, 81 (2007), 1-3: 29-82.

[3] de Laguna, T., "Point, line and surface as sets of solids" The Journal of Philosophy, 19 (1922), 17: 449-461.

[4] Dimov, G., and D. Vakarelov, "Contact algebras and region-based theory of space. A proximity approach. I and II", Fundamenta Informaticae, 74 (2006), 2-3: 209-249, 251-282.

[5] Düntsch, I., and M. Winter, "A representation theorem for Boolean contact algebras", Theoretical Computer Science (B), 347 (2005), 3: 498-512. 
[6] Egenhofer, M., and R. Franzosa, "Point-set topological spatial relations", International Journal of Geographical Information Systems, 5 (1991), 2: 161-174.

[7] Ershov, Y. L., Problems of Decidability and Constructive Models (in Russian), Science, Moskow, 1980.

[8] Finger, M., and D. M. Gabbay, "Adding a temporal dimension to a logic system", Journal of Logic, Language and Information, 1 (1992), 3: 203233.

[9] Jonsson, P., and T. Drakengren, "A complete classification of tractability in the spatial theory RCC-5", Journal of Artificial Intelligence Research, 6 (1997): 211-221.

[10] Kontchakov, R., A. Kurucz, F. Wolter, and M. Zakharyaschev, "Spatial logic + temporal logic = ?", in M. Aiello, I. Pratt-Hartmann, and J. van Benthem (eds.), Handbook of Spatial Logics, chapter 9, Springer, 2007, pp. $497-564$.

[11] Lutz, C., and F. Wolter, "Modal logics for topological relations", Logical Methods in Computer Science 2, 2-5 (2006): 1-41.

[12] Nenchev, V., "Logics for stable and unstable mereological relations", Central European Journal of Mathematics, 9 (2011), 6: 1354-1379.

[13] Nenchev, V., "Undecidability of logics for mereological and mereotopological relations", in Proceedings of $8^{\text {-th }}$ Panhellenic Logic Symposium, Ioannina, Greece, July 2011.

[14] Nenov, Y., and D. Vakarelov, "Modal logics for mereotopological relations", in C. Areces and R. Goldblatt (eds.), Advances in Modal Logic, Nancy, France, September 2008, pp. 249-272.

[15] Pratt-Hartmann, I., "First-order mereotopology", in M. Aiello, I. PrattHartmann and J. van Benthem (eds.), Handbook of Spatial Logics, chapter 2, Springer, 2007, pp. 13-97.

[16] Randell, D. A., Zhan Cui, and A.G. Cohn, "A spatial logic based on regions and connection", in B. Nebel, C. Rich, and W. R. Swartout (eds.), Proceedings of $3^{\text {rd }}$ International Conference Knowledge Representation and Reasoning, Cambridge, Massachusetts, USA, October 1992, Morgan Kaufmann, pp. 165-176.

[17] Simons, P., Parts: A Study in Ontology, Oxford University Press, 1987.

[18] Stell, J. G., "Boolean connection algebras: A new approach to the region connection calculus", Artificial Intelligence, 122 (2000), 1-2: 111-136.

[19] Vakarelov, D., "Logical analysis of positive and negative similarity relations in property systems", in M. De Glas and D. Gabbay (eds), Proceedings of $1^{-s t}$ World Conference on the Fundamentals of Artificial Intelligence, Paris, France, July 1991, pp. 491-499. 
[20] Vakarelov, D., "A modal logic for set relations", in Proceedings of $10^{\text {th }}$ International Congress of Logic, Methodology and Philosophy of Science, Abstracts, Florence, Italy, August 1995, p. 183.

[21] Vakarelov, D., "Region-based theory of space: Algebras of regions, representation theory and logics", in D. M. Gabbay, M. Zakharyaschev, and S. S. Goncharov (eds.), Mathematical Problems from Applied Logics II. Logics for the XXIst Century, Springer, 2007, pp. 267-348.

[22] Vakarelov, D., "A modal approach to dynamic ontology: modal mereotopology", Logic and Logical Philosophy, 17 (2008): 167-187.

[23] Vakarelov, D., "Dynamic mereotopology: A point-free theory of changing regions. I. Stable and unstable mereotopological relations", Fundamenta Informaticae, 100 (2010), 1-4: 159-180.

[24] Whitehead, A. N., Process and Reality, New York: MacMillan, 1929.

[25] Wolter. F., and M. Zakharyaschev, "Spatio-temporal representation and reasoning based on RCC-8", in A. G. Cohn, F. Giunchiglia, and B. Selman (eds.), Proceedings of the rth $^{\text {th }}$ Conference on Principles of Knowledge Representation and Reasoning, Breckenridge, Colorado, USA, April 2000, Morgan Kaufmann, pp. 3-14.

\section{VLADISLAV NENCHEV}

Department of Mathematical Logic and Applications

Faculty of Mathematics and Informatics

Sofia University "St. Kl. Ohridski"

5 James Bourchier blvd.

Sofia, 1164, Bulgaria

lucifer.dev.0@gmail.com 